UCRL-JC-123551

PREPRINT

\title{
Deflection, Spraying and Induced Scattering of Intense Laser Beams in Plasmas
}

\author{
W. L. Kruer
}

\section{PECENGE \\ OCT 211996 \\ OSTI}

This paper was prepared for submittal to the 1996 International Conference on Plasma Physics

Nagoya, Japan

September 9-13, 1996

September 4, 1996

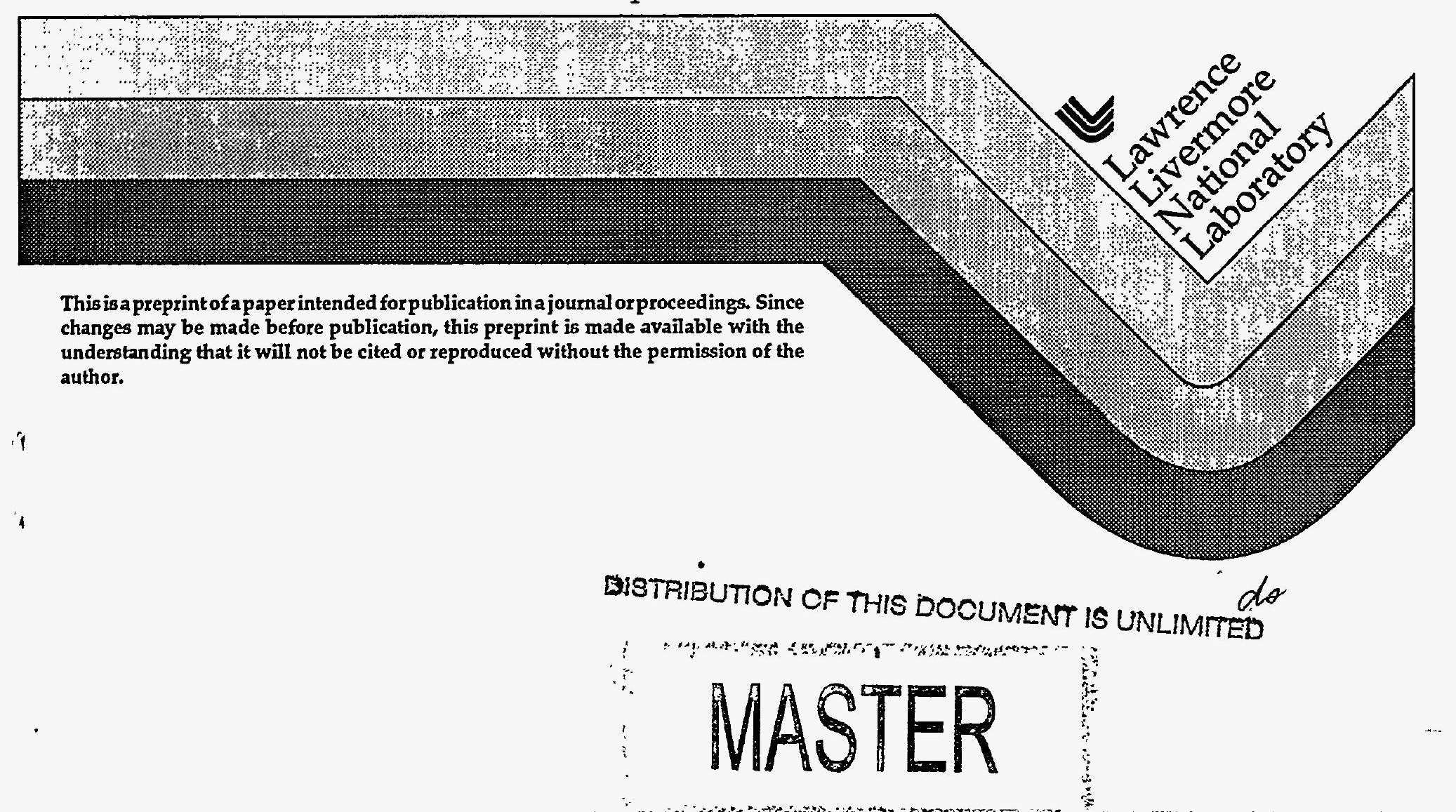




\section{DISCLAIMER}

This document was prepared as an account of work sponsored by an agency of the United States Government. Neither the United States Government nor the University of California nor any of their employees, makes any warranty, express or implied, or assumes any legal liability or responsibility for the accuracy, completeness, or usefulness of any information, apparatus, product, or process disclosed, or represents that its use would not infringe privately owned rights. Reference herein to any specific commercial product, process, or service by trade name, trademark, manufacturer, or otherwise, does not necessarily constitute or imply its endorsement, recommendation, or favoring by the United States Government or the University of California. The views and opinions of authors expressed herein do not necessarily state or reflect those of the United States Government or the University of California, and shall not be used for advertising or product endorsement purposes. 
XI 96-125

Deflection, Spraying and Induced Scattering of Intense Laser Beams in Plasmas

\author{
William L. Kruer
}

Lawrence Livermore National Laboratory

\begin{abstract}
Investigations into laser beam spraying, deflection, and induced scattering in plasmas are presented. Recent calculations and experiments on beam spraying due to filamentation are discussed. A simple model is presented for an enhanced beam deflection associated with nearly sonic plasma flow transverse to the beam. This model provides useful insights on the laser beam deflection, its scaling and the importance of self-consistent profile modifications. Finally, some discussion is given of recent experiments demonstrating the interplay between stimulated Raman and Brillouin scattering.
\end{abstract}

\title{
1. Introduction
}

An improved understanding of laser plasma interactions is important for inertial fusion as well as for numerous advanced applications such as laser plasma accelerators. Laser plasma interactions are also a challenging test bed for the understanding of many basic plasma phenomena. An intense light wave in a plasma can drive a number of instabilities ${ }^{1}$, such as stimulated Raman and Brillouin scattering as well as ponderomotive or thermal filamentation. The stimulated scattering instabilities can be most simply characterized as the resonant decay of the intense lightwave into a scattered light wave plus either an ion 
acoustic wave (SBS) or an electron plasma wave (SRS). Filamentation represents the growth of intensity modulations in a laser beam associated with either ponderomotively or thermally-driven density modulations transverse to the beam. In the conventional approach ${ }^{2}$ to inertial fusion, it is desirable to maximize collisional absorption (inverse bremsstrahlung) and minimize instabilities and other plasma effects which can reduce the absorption, change its location, or produce energetic electrons which preheat the capsule.

Here we discuss some recent research directed to understanding and controlling laser beam filamentation, laser beam deflection due to plasma flow transverse to the beam, and the cross talk between stimulated Raman and Brillouin scattering.

\section{Angular divergence and filamentation}

Progress has been made in understanding and controlling laser beam filamentation. This filamentation occurs because a region of increased laser intensity locally depresses the plasma density either via the ponderomotive force or the thermal force (i.e., the pressure increase associated with the.higher temperature). The depression in plasma density acts to focus the light, thereby increasing the intensity fluctuation. Filamentation describes the unstable growth of infinitsimal modulations. In practice, there are significant hot spots in the laser beam, and the physical process is more appropriately characterized as hot spot self-focusing.

Filamentation and hot spot self-focusing of laser light can be suppressed by introduction of spatial incoherence, say, by use of a random plate plate. ${ }^{3}$ An angular spread in wave vectors is thereby created. The wave vectors are spread into a cone with a characteristic half-angle $A \Theta$ about the original wave vector of 


\section{DISCLAIMER}

Portions of this document may be illegible in electronic image products. Images are produced from the best available original document. 
the light wave. This angular divergence ${ }^{3-7}$ clearly acts to counter nonlinear focusing. A crude estimate 4 is that the instability is suppressed when the correlation length in the transverse directions $\left(\ell \perp \cong \frac{2 \pi}{k_{0} \Delta \Theta}\right)$ is less than the wavelength of the most unstable filaments. If we consider ponderomotive filamentation, this condition becomes

$$
(\Delta \Theta)^{2} \geq \frac{1}{4} \frac{\omega_{\mathrm{pe}}^{2}}{\omega_{\mathrm{o}}^{2}} \frac{\mathrm{v}_{\mathrm{os}}^{2}}{\mathrm{v}_{\mathrm{c}}^{2}}
$$

where $\omega_{\mathrm{pe}}$ is the electron plasma frequency, $\omega_{0}$ the laser light frequency, $v_{\mathrm{os}}$, the oscillation velocity of an electron in the laser field and $v_{e}$ the electron thermal velocity. The same scaling can be obtained in other ways, such as requiring that the coherence length in the direction of propagation be less than either a filamentation growth length or the self-focusing length of a hot spot. It should be noted that temporal incoherence ${ }^{7}$ also helps to suppress filamentation. A small bandwidth smooths the spatial interference patterns on a time scale less than the filament growth time.

Beam divergence can also be introduced by the interaction of a laser beam with density fluctuations in the plasma. Indeed, small amplitude density modulations in the transverse direction with wavelength $\lambda_{1} \ll \lambda_{\max }$ (the wavelength of the most unstable mode) can suppress filamentation. 4 A density fluctuation with amplitude $\delta$ couples an incident wave into a light wave at an angle $\left(\theta \cong \lambda_{\perp} / \lambda_{0}\right)$ in a distance of $k_{0} z \sim\left(\frac{1}{2} \frac{\delta n}{n_{c c}}\right)^{-1}$. This distance is less than a growth length when $\frac{\delta n}{n} \geq \frac{1}{4}\left(\frac{v_{\alpha s}}{v_{c}}\right)^{2}$, which for typical parameters corresponds to a small amplitude. This suppression has been observed in simulations of ponderomotive filamentation using ${ }^{9} 3 \mathrm{D}$ model for light wave propagation. 
These results suggest the possibility of plasma-induced beam smoothing. One scheme is to send along a low intensity seed beam which has an angular spread and a small bandwidth (of order $0.1 \%$ ). The aim is to induce Brillouin near forward scattering of the main beam, which is thereby smoothed.

In turn, filamentation can induce angular divergence in a laser beam. This spraying of a laser beam as it strongly filaments has been illustrated in computer simulations ${ }^{8}$ of ponderomotive filamenatation by S. Wilks using the F3D code. 5 Figure 1 shows calculated intensity contours of the electric field of the $1.06 \mu \mathrm{m}$ Gaussiam beam with an initial peak intensity of $2 \times 10^{15} \frac{\mathrm{W}}{\mathrm{cm}^{2}}$. In this simulation the beam is incident onto a plasma with a parabolic density profile and a peak density of $0.2 n_{\mathrm{cr}}$ (the critical density), an electron temperature of $1 \mathrm{keV}$, and an ion temperature of $0.5 \mathrm{keV}$. As the laser beam filaments, it sprays out in angle. The characteristic spread is $\Delta \Theta \cong \frac{1}{2} \frac{\omega_{\mathrm{pe}}}{\omega_{0}} \frac{v_{0 s}}{v_{c}}$. The scaling can again be motivated in various ways. Note the angular divergence nonlinearly-induced is that which would have suppressed filamentation of the incident beam, as estimated in Equation 1.

The spraying of the laser beam correlated with the onset of filamentation has been șhown in experiments. 8.9 In these experiments with the 2 beam $1.06 \mu \mathrm{m}$ Janus laser, a plasma was preformed by using one beam to irradiate a thin foil. A second 100ps interaction beam was delayed so that it interacts with an underdense, preformed plasma with various peak densities. By the use of film around an $f / 2$ collecting lens, the angular spraying of the interaction beam was directly monitored. As the peak plasma density was increased from $0.06 n_{c r}$ to $0.12 n_{c r}$, (lowering the threshold for filamentation), light was indeed observed to spread to larger angles, consistent with the predictions. It should be noted that near forward 
Brillouin scattering can also contribute 10 to angular spraying, depending on the efficiency of this process and its sensitivity to velocity gradients.

\section{Laser beam deflection in nonlinearly generated flow profiles}

An improved understanding of how intense laser beams are deflected in plasmas is important for many applications. For example, in hohlraums filled with low density plasma, laser beams are observed ${ }^{11}$ to be deflected in angle. Beam pointing must then be changed to recover symmetric implosions. In addition, an intensity-dependent deflection has been observed in recent experiments ${ }^{12}, 13$ with preformed plasmas.

A recently proposed explanation 14,15 for the beam bending invokes filamentation in a plasma with a flow transverse to the beam. As just discussed, filamentation can induce an angular spread in a laser beam. If the symmetry is broken by, say, a transverse plasma flow, this spreading can become asymmetrical; i.e., a mean deflection can occur.

The linear theory of ponderomotive filamentation in a flowing plasma has been discussed by Short et al. 18 The usual dispersion relation now becomes

$$
\frac{\mathrm{K}^{2}}{\mathrm{k}_{\perp}^{2}}=\frac{\mathrm{n}}{8 \mathrm{n}_{\mathrm{cs}}}\left(\frac{\mathrm{v}_{o s}}{\mathrm{v}_{\mathrm{e}}}\right)^{2} /\left(1-\mathrm{v}_{\perp}^{2} / \mathrm{c}_{\mathrm{s}}^{2}\right)-\frac{\mathrm{k}_{\perp}^{2}}{4 \mathrm{k}_{\mathrm{o}}^{2}} \text {. }
$$

Here $\mathrm{K}$ is the spatial gain rate, $\mathrm{k}_{\perp}$ the transverse wave number, $\mathrm{v}_{\perp}$ the transverse flow, $c_{s}$ the ion sound speed, $k_{0}$ the wave number of the laser light, $v_{0 s}$ the oscillation velocity of an electron in the electric field, $v_{e}$ the electron thermal velocity, $n$ the plasma density, and $n_{\mathrm{cr}}$ the critical density. Equation (1) assumes that $\mathrm{K} \ll \mathrm{k}_{\perp}$. It is apparent that the growth rate increases (threshold decreases) as $v_{\perp}$ approaches $c_{s}$. For $v_{\perp} \cong c_{3}, K \sim k_{\perp}$ and one must solve the full dispersion relation, finding that maximum growth occurs for filaments which grow at an angle to the direction of light propagation. 
In general, deflection of a laser beam only depends on there being induced changes in the plasma density profile. Useful insights can be obtained by considering a simple model of a laser beam propagating transverse to a freelyexpanding plasma near its sonic point. ${ }^{17}$ We first estimate the nonlinearly steepened profile and then calculate the laser beam deflection in this profile. Filamentation can modify the profile changes and beam deflection, although it should be emphasized that the "zero order" profile changes help stabilize filamentation.

As a simple model, let's consider a one-dimensional expanding plasma traversed by a laser beam with its propagation vector orthogonal to the gradient of the plasma density and expansion velocity. The beam radius is assumed to be much less than the plasma scale lengths, which are in turn sufficiently long to allow neglect of refraction in the unperturbed profiles. The beam traverses a region of plasma which includes the sonic point. We anticipate that the plasma profile will be locally steepened in the neighborhood of the sonic density $\left(n_{s}\right.$, where $\left.u=c_{s}\right)$ from a density $n_{2}>n_{s}$ to a density $n_{1}<n_{2}$. If we use the 2-fluid model in a frame moving with the critical surface, 18

$$
\begin{aligned}
& \frac{\partial}{\partial x} n u=0 \\
& \frac{\partial}{\partial x} \frac{u^{2}}{2}=-c_{3}^{2} \frac{\partial}{\partial x} \ln n-\frac{Z m}{4 M} \frac{\partial}{\partial x} v_{w}^{2}
\end{aligned}
$$

Here $n$ is the plasma density, $u$ the flow velocity, $c_{s}$ the ion sound speed, $Z$ the ion charge state, $m(M)$ the electron (ion) mass, and $v_{W}$ the oscillation velocity of an electron in the electric field of the laser light. For $\frac{\mathbf{v}_{o s}}{v_{c}} \ll 1$, we find that the profile is steepened over a density $\Delta n \equiv n_{s}\left(\frac{V_{o s}}{V_{c}}\right)$. in a distance of about $2 r_{0}$, where $r_{0}$ is the 
beam radius. Note that $\Delta \mathrm{n}$ is proportional to the square root of the laser intensity, since the plasma is resonantly perturbed near the sonic point.

The deflection of the laser beam by refraction in the locally steepened profile is now readily estimated. The equation for a light ray is

$$
\ddot{\xi}=\frac{-c^{2}}{2 n_{c s}} \nabla n
$$

where $\xi$ is the ray displacement, $c$ the velocity of light, $n$ the plasma density, and $\mathrm{n}_{\mathrm{cr}}$ the critical density. Approximately $\nabla \mathrm{n} \cong \mathrm{n} \frac{\mathrm{v}_{\mathrm{os}}}{\mathrm{v}_{\mathrm{e}}} \mathrm{e}^{-0.25} / 2 \mathrm{r}_{\mathrm{o}}$, we estimate the deflection angle is

$$
\theta \equiv \frac{\ell}{4 \mathrm{r}_{\mathrm{o}}} \frac{\mathrm{n}}{\mathrm{n}_{\mathrm{cr}}} \frac{\mathrm{v}_{\mathrm{os}}}{\mathrm{v}_{\mathrm{e}}} \mathrm{e}^{-0.25},
$$

where $\ell=c t$ is approximately the distance traveled by the laser beam.

In general, $\ell$ can be determined by plasma inhomogeneity. One ideal example is a shear ${ }^{15}$ in the transverse flow velocity along the beam path. As is apparent from Equations 7 and 8, the flow is only resonantly perturbed over a distance $\ell \cong \ell_{v} \frac{v_{o s}}{v_{c}}$, where $\ell_{v}$ is the shear velocity gradient length. For a laser beam (or hot spot) with half-width $r_{0}$, we then estimate $\theta \cong \frac{\ell_{v}}{4 r_{0}} \frac{n}{n_{c x}}\left(\frac{v_{o s}{ }^{2}}{v_{c}^{2}}\right)$ Note that in this limit, one should probably also consider the net contribution of the plasma which is non-resonantly perturbed (i.e., where the induced profile changes scales as $\left(\frac{v_{\alpha o}}{v_{c}}\right)^{2}$ ). For a $0.35 \mu m$ laser beam with a half-width of $20 \lambda 0$ and an intensity of $4 \times 10^{15} \frac{\mathrm{W}}{\mathrm{cm}^{2}}$ in the plasma considered above and for $\ell_{\mathrm{v}} \cong 1500 \lambda_{0}, \theta \cong 9^{\circ}$.

Some estimates are enlightening. As shown by Equation 1, the threshold for filamentation can be greatly reduced near the sonic point. However, the selfconsistent profile changes prevent large reductions unless $\frac{v_{\text {os }}}{v_{e}}$ is quite small. A simple physical estimate is illustrative. Since the velocity jumps by $\left(\frac{v_{o s}}{v_{e}}\right) c_{s}$ in the 
modified zeroth-order state, $\min \left(1-\frac{v_{1}^{2}}{c_{s}^{2}}\right) \min \sim 2 \frac{v_{\alpha}}{v_{c}}$. Hence, the threshold is reduced for inertial fusion parameters by about $\sqrt{\frac{2 v_{o s}}{v_{c}}} \cong 0.5$, only a modest reduction. Strong ion wave damping can also prevent strong reductions in threshold.

\section{The competition between SRS and SBS}

Finally, there can be a rich interplay between the stimulated Raman and Brillouin instabilities. For example, what is done to minimize SBS can lead to an increase in SRS.19-21 As an example of the rich interplay, let's first consider some recent work on reducing SBS by enhancing the ion wave damping and then discuss how enhanced ion wave damping can increase SRS.

In large, nearly homogeneous plasmas, SBS can be reduced by adding hydrogen to the plasma. Linear theory 22 predicts that the light protons enhance the Landau damping of the ion waves associated with SBS. Simulations ${ }^{23,24}$ confirm this enhanced damping and show a reduction of SBS reflectivity even in the nonlinear state. This reduction of reflectivity by addition of protons has been demonstrated in experiments 25,26 using the Nova laser with both gas bag and hohlraum targets. In the experiments, plasmas with a size of about $2 \mathrm{~mm}$, an electron plasma density of about $10^{21} \mathrm{~cm}^{-3}$, and an electron temperature about $3 \mathrm{keV}$ are formed by irradiating gas bag targets with nine Nova beams. Figure 2a shows the measured SBS back reflection of a tenth, interaction beam as a function of plasma composition (the percentage of protons). This beam was focused with an $\mathrm{f} / 4.3$ lens to an intensity of about $2 \times 10^{15} \frac{\mathrm{W}}{\mathrm{cm}^{2}}$. Note the strong decrease in reflectivity as the proton percentage (by number) increases. Figure $2 \mathrm{~b}$ shows the similar trend found in some representative simulations using a particle ion, fluid 
electron code. Similar results were found in early experiments in which low temperature and density discharge plasmas were irradiated with $\mathrm{CO}_{2}$ laser light. ${ }^{27}$

However, there are regimes in which increased ion wave damping can lead to more SRS. This behavior has been shown in recent experiments ${ }^{19-21}$ using both gas bag and hohlraum targets. As an example, consider experiments ${ }^{19}$ using the Nova Laser and gas bag targets. In Figure 3, the peak SRS, back reflection is plotted versus the ion wave damping. This damping was varied by changing the concentration of a $\mathrm{C}_{5} \mathrm{H}_{12}$ impurity in a Xe plasma. The electron plasma density was about $9 \times 10^{20} \mathrm{~cm}^{-3}$, and the SSD-smoothed $0.35 \mu \mathrm{m}$ interaction beam $(f / 4.3)$ had an intensity of about $7 \times 10^{15} \frac{\mathrm{W}}{\mathrm{cm}^{2}}$. In the early time results, the heater beams which formed the plasma are still on, leading to a hotter plasma ( $3 \mathrm{keV})$ than in the so-called late time results. Note that there is a regime in which the SRS reflectivity increases as the nominal ion wave damping increases. Note also that with the higher temperature plasma most relevant to inertial fusion, the SRS reflectivity does flatten at $\sim 5 \%$.

This behavior has been predicted by models $28-31$ which invoke saturation of SRS by parametric decay of the Raman-driven plasma wave into ion waves and other electron plasma waves. This subsequent decay has a threshold

$$
\left(\frac{\delta \mathrm{n}}{\mathrm{n}}\right)^{2} \sim 4 \mathrm{k}^{2} \lambda_{\mathrm{Dc}}^{2} \frac{\mathrm{v}_{i}}{\omega_{\mathrm{i}}} \frac{\mathrm{v}_{e}}{\omega_{\mathrm{pc}}}
$$

where $\delta \mathrm{n}$ is the fluctuation plasma density associated with the Raman-driven plasma wave, $k$ its wavenumber and De the electron Debye length. Here $v_{i}\left(\omega_{i}\right)$ is the ion wave energy damping rate (frequency) and $v_{e}\left(\omega_{\mathrm{pe}}\right)$ is the electron plasma wave damping rate (frequency). As the ion wave damping rate increases, the Raman-generated plasma wave can grow to higher levels, giving larger scattering. A similar scaling might be obtained from saturation via a decay of the Raman plasma wave into a scattered light wave ${ }^{31}$ plus an ion wave. 
In summary, some recent investigations of the spraying, deflection and induced scattering of an intense laser beam have been discussed. Angular divergence both suppresses and nonlinearly results from laser beam filamentation. Near sonic plasma flow transverse to the beam leads to an enhanced deflection. Finally, a rich interplay can take place between stimulated Raman and Billouin scattering, as illustrated by their nonlinear behavior when the ion wave damping is varied. Laser plasma interactions clearly continue to be a challenging test bed for understanding plasma behavior.

\section{Acknowledgments}

I am grateful for numerous collaborations on the various topics of this paper, as detailed in the references.

*Work performed under the auspices of the U.S. Department of Energy by the Lawrence Livermore National Laboratory under Contract W-7405-Eng-48. 


\section{References}

[1] Baldis H A, Campbell E M and Kruer W L, Physics of Laser Plasmas (North Holland, Amsterdam, 1991) 361-434

[2] Lindl J 1995 Phys. of Plasmas 23933

[3] Kato Y, Mima K, Arinaga S, Kitagawa Y, Natzatsoka M and Yamanaka C 1982 Phys. Rev. Lett. 53, 1057

[4] Kruer W L, Laser Interaction and Related Plasma Phenomena (Plenum, New York, 1993) 10,503

[5] Berger R L, Lasinski B F, Kaiser T B, Williams E A, Langdon A B and Cohen B I 1993 Phys. Fluids B5, 2243

[6] Rose H A and Dubois D F 1992 ibid. 42521

[7] Schmitt A J 1988 Phys. Fluids 313079

[8] Wilks S, Young P E, Hammer J, Tabak M and Kruer W L 1994 Phys. Rev. Letters 732994

[9] Young P E, Hammer J H, Wilks S C and Kruer W L 1995 Phys. Plasmas 22825

[10] Rozmus W 1996 Private Communication

[11] Glendinning S G, Powers L V, Kauffman R L, Landen O L, Ress B B, Stone G F, Suter L J and Richard A L 1995 submitted to Phys Rev. Letters

[12\} Moody J D, MacGowan B J, Hinkel D E, Kruer W L, Williams E A, Estabrook K G, Shepard T D, Kirkwood R, Montgomery D S and Berger R L 1996 Phys. Rev. Letters 
[13] Bauer B et al to be published

[14] Rose H A 1995 Phys. Plasmas 31709

[15] Hinkel D E, Williams E A and Still C H 1996 Phys. Rev. Letters

[16] Short R W, Bingham R and Williams E A 1982 Phys. Fluids 252392

[17] Kruer W L and Hammer J H in press Comments Plasma Phys. Controlled Fusion

[18] Lee K, Forslund D W, Kindel J M and Lindman E L 1977 Phys. Fluids 2051

[19] Kirkwood R K, MacGowan B J, Montgomery D S, Afeyan B B, Kruer W L, Moody J D, Estabrook K G, Back C A, Glenzer SH, Blain M A, Williams E A, Berger R L and Lasinki B F September 1996 Phys. Rev. Letters

[20] Fernandez J C, Cobble J A, Failor B H, DuBois D F, Montgomery D S, Rose H A, Vu H X, Wilde B H, Wilke M D and Chrien R E September 1996 Phys. Rev. Letters

[21] Montgomery D S, MacGowan B J, Kirkwood R K, Moody J D, Stone G F, Afeyan B B, Berger R L, Estabrook K G, Kruer W L, Lasinski B F, Munro D H and Williams E A submitted to Phys. Rev. Letters

[22] Vu H X et al 1994 Phys. Plasmas 13542; Williams E A 1995 et a ibid 2129

[23] Wilks S C, Kruer W L, Denavit J, Estabrook K, Hinkel D E, Kalantar D, Langdon A B, MacGowan B, Montgomery D S and Williams E A 1995 Phys. Rev. Letters 7425

[24] Vu H X J. Comp. Phys.

[25] MacGowan B et al 1996 Lawrence Livermore National Laboratory UCRL-LR105821-95-4 305-331 
[26] Fernandez J C et al 1996 Phys. Rev. E 532747

[27] Clayton C E, Joshi C, Yasuda A and Chen F F 1981 Phys. Fluids 242312

[28] Karttunen SJ 1981 Phys. Rev. A 23 2006; Drake R P and Batha S H 1991 Phys. Fluids B 32936

[29] Bezzerides B, DuBois D F and Rose H A 1993 Phys Rev. Letters 702569

[30] Kolber J T, Rozmus W and Tikhonchuk V T 1993 Phys Fluids B 5138

[31] Baker KL 1996 Ph.D. Dissertation Univeristy of California Davis 
Figure captions:

Figure 1. A plot of calculated intensity contour of the electric field of a $1.06 \mu \mathrm{m}$ laser beam propagating through a plasma with a parabolic density profile.

Figure 2a. The SBS reflectivity measured with gas bag targets versus the percentage of protons in the plasma.

Figure $2 \mathrm{~b}$. The drop-off in SBS reflectivity as a percentage of protons in the plasma as calculated with a hybrid particle-ion fluid-electron code.

Figure 3. The SRS reflectivity measured with gas bag targets versus the impurity fraction and normalized ion wave damping. 


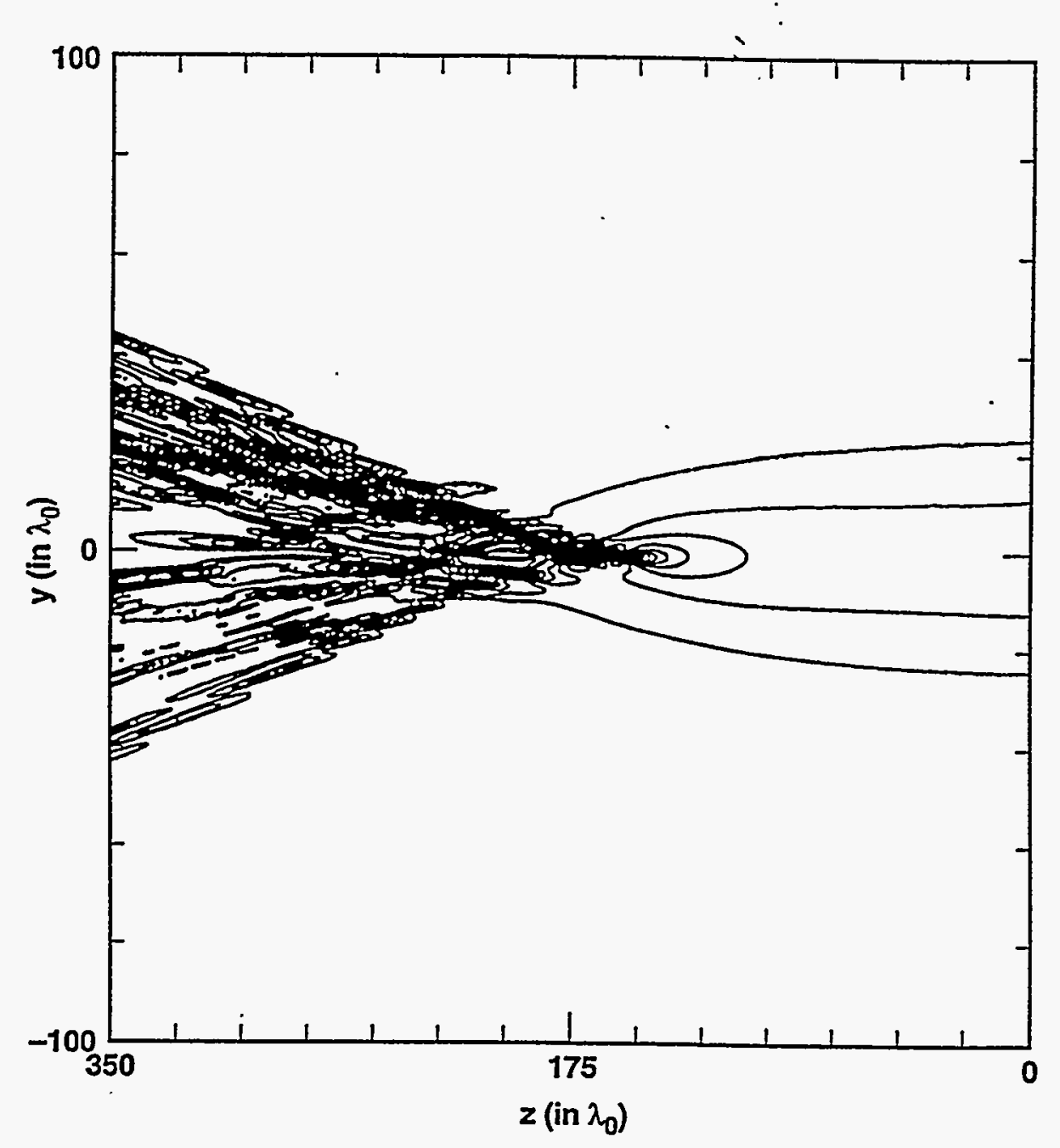

50-00-0896-1982pb01

$26 \mathrm{BK} / \mathrm{mem}$ 
(a) SBS reflectivity measured

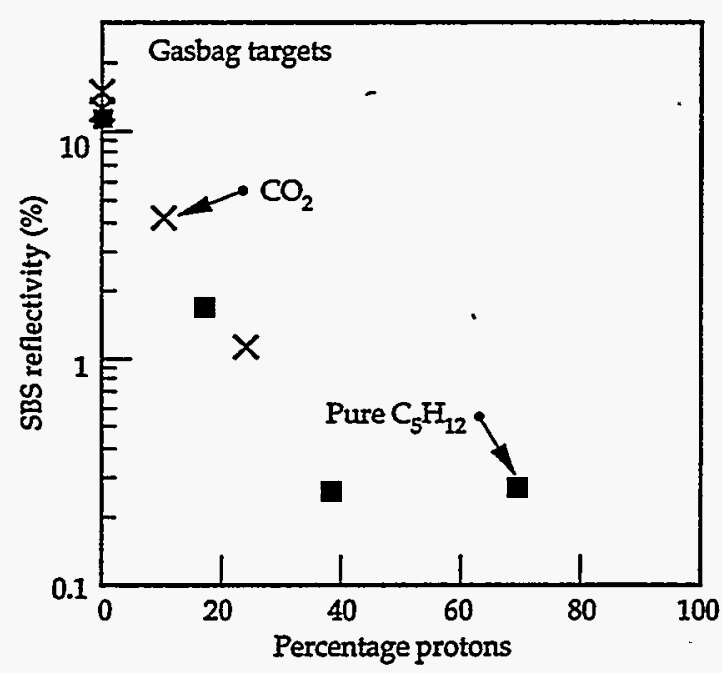

(b) PIC-calculated reflectivity

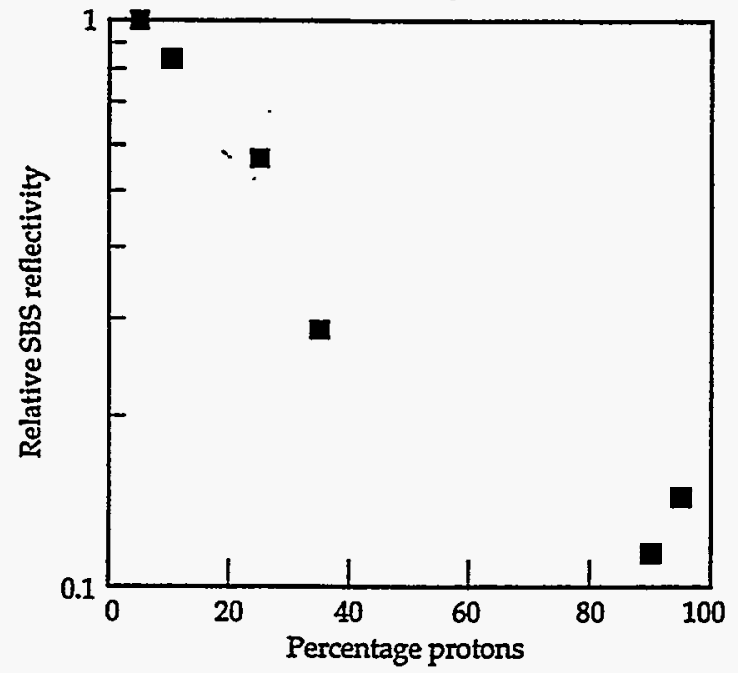




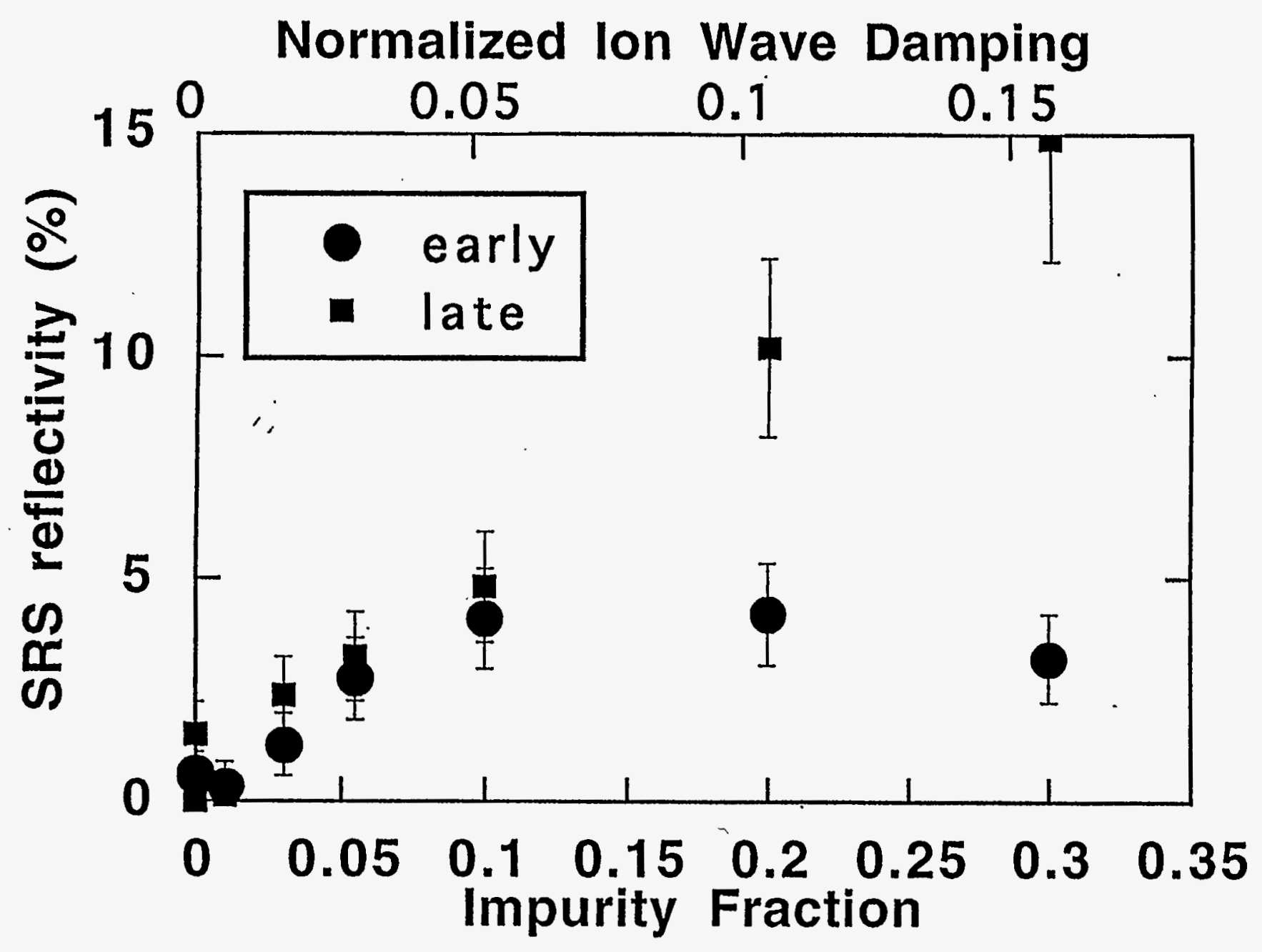

